\title{
Importância da Atuação do Psicólogo no Tratamento de Mulheres com Câncer de Mama
}

\author{
The Importance of Psychological Interventions in the Treatment of Women \\ with Breast Cancer
}

Juliana Lima Venâncio* e Vera Maria Stiebler Leal

\begin{abstract}
Resumo
D ecorrente da grande incidência do câncer de mama no Brasil e da desestruturação que esse diagnóstico e tratamento acarretam na vida da mulher, é fundamental a atuação do psicólogo para dar suporte à paciente e a seus familiares nesse momento. Esse profissional vai ajudar a mulher a manter o seu bem-estar psicológico durante 0 tratamento. Vários estudos referentes ao câncer de mama comprovam que pacientes que participam de atendimento psicológico possuem um melhor ajustamento à doença, redução dos distúrbios emocionais (como ansiedade e depressão), melhor adesão ao tratamento e diminuição dos sintomas adversos associados ao câncer e aos tratamentos, podendo até obter um aumento no tempo de sobrevida. A atuação do psicólogo neste campo é muito recente e pouco conhecida. Acreditamos ser importante a divulgação da relevância desta especialidade a fim de obter reconhecimento por parte dos profissionais de saúde, criando assim uma maior demanda para o psicólogo.

Palavras-chave: adaptação psicológica; neoplasias mamárias; saúde da mulher; psicologia; pacientes; qualidade de vida.

Abstract

Considering the large incidence of breast cancer in Brazil and the problems involving the diagnosis and treatment in the lives of the women with breast cancer, it is very important the work of psychotherapists, who help and give all the proper support to the patient and their family during that tough period. This professional will help women on maintaining their psychological well-being during the treatment. A full range of studies on breast cancer confirms that all the patients who have undergone a psychological treatment have a better adjustment to the disease, less emotional distresses (like anxiety and depression), a better response to the treatment and a significant reduction of the adverse symptoms caused by the cancer itself and its treatment, and the life span of the patient may be even increased. The work of psychotherapists in the field is very recent and still not well understood. We believe that it is very important and extremely necessary for this discipline to be recognized and properly disseminated among health professionals, so that the patients get the psychological and emotional support they need.
\end{abstract}

Key words: psychological adaptation; breast neoplasms; women's health; psychology; patients; quality of life.

\footnotetext{
* Especialista em Psicologia M édica - H UPE/ UERJ. Especialista em Psicologia O ncológica - IN CA

Trabalho apresentado ao Instituto $\mathrm{N}$ acional de Câncer para a obtenção do título de Especialista em Psicologia O ncológica.

Endereço para correspondência: Juliana Lima Venâncio - Av. Afrânio de M elo Franco 42 apt. 502 - Leblon - Rio de Janeiro CEP: $22430-060$ - email: julvenancio@terra.com.br
} 


\section{INTRODUÇÃO}

Apesar dos avanços da medicina no tratamento do câncer e do aumento de informações veiculadas pela mídia, o câncer ainda equivale, muitas vezes, a uma "sentença de morte", comumente associado a dor, sofrimento e degradaçãa ${ }^{1,2}$. A literatura nos mostra que o câncer sempre foi percebido como algo vergonhoso, sujo, contagioso e sem cura, sendo uma doença tradicionalmente relegada pela sociedade ${ }^{3,4}$.

0 diagnóstico de câncer e todo o processo da doença são vividos pelo paciente e pela sua família como um momento de intensa angústia, sofrimento e ansiedade. Além do rótulo de uma doença dolorosa e mortal, o paciente comumente vivencia no tratamento, geralmente longo, perdas e sintomas adversos, acarretando prejuízos nas habilidades funcionais, vocacionais e incerteza quanto ao futuro ${ }^{5,6}$. Muitas fantasias e preocupações em relação à morte, mutilações e dor encontram-se presentes.

No câncer de mama, além das preocupações citadas acima, encontram-se presentes outras angústias ligadas à feminilidade, maternidade e sexualidade, já que o seio é um órgão repleto de simbolismo para a mulher. Sendo assim, o anúncio desse diagnóstico, seguido pelos tratamentos, pode ocasionar abalos significativos na vida da paciente.

Entre as mulheres, o câncer de mama apresenta-se como a segunda neoplasia maligna com maior incidência, assim como uma causa relevante de mortes por câncer no Brasil. D essa forma, essa patologia é considerada um dos maiores problemas de saúde pública associado ao câncer em mulheres nesse país ${ }^{7-10}$. No entanto, é um tipo de câncer que possui uma significativa possibilidade de sobrevida quando detectado precocemente ${ }^{11}$.

Um importante fator que tem contribuído para o aumento da sobrevida das mulheres com câncer de mama são os avanços da medicina ${ }^{4}$, proporcionando tratamentos mais eficientes e técnicas que viabilizam a detecção precoce. Com esses progressos terapêuticos, o câncer vai deixando de ser uma doença freqüentemente fatal e assumindo características de uma doença crônica ${ }^{12}$.

Considerando-se a alta incidência do câncer de mama, a grande possibilidade de uma longa sobrevida e a desestruturação que o diagnóstico e tratamento do câncer de mama acarretam na vida da mulher, tem ocorrido uma maior demanda para se investir na qualidade de vida da paciente ${ }^{13,14}$.

Qualidade de vida é aqui definida como "um conceito multidimensional que é mensurado como uma avaliação subjetiva do status de saúde e do bem-estar em diferentes setores da vida, incluindo componentes físicos e psíquicos"15. Dessa forma, a atuação do psicólogo é fundamental ao longo do tratamento, já que sua prática visa o bem-estar emocional da paciente, contribuindo assim para uma boa qualidade de vida.

\section{IMPACTO NA VIDA DA MULHER COM CÂNCER DE MAMA: CONSEQÜÊNCIAS EMOCIONAIS DO DIAGNÓSTICO E TRATAMENTO}

A eclosão do câncer de mama na vida da mulher acarreta efeitos traumáticos, para além da própria enfermidade. $A$ mulher se depara com a iminência da perda de um órgão altamente investido de representações, assim como o temor de ter uma doença sem cura, repleta de sofrimentos e estigmas.

A partir do diagnóstico confirmado, a paciente vê sua vida tomar um rumo diferente do que poderia imaginar, já que o câncer pode acarretar alterações significativas nas diversas esferas da vida como trabalho, família e lazer $^{13}$. D essa forma, acaba trazendo implicações em seu cotidiano e nas relações com as pessoas do seu contexto social6.

Sales et al. ${ }^{13}$ ao pesquisarem a qualidade de vida das mulheres tratadas de câncer de mama à luz do funcionamento social, evidenciam que as mudanças no trabalho, lazer, relações familiares e sociais dessas mulheres são provocadas mais por problemas psicológicos do que físicos.

Essa realidade que impera faz com que a paciente e seus familiares assumam papéis que não foram escolhidos e sim impostos pela fatalidade do adoecimento, interrompendo planos, ideais e perspectivas futuras. A constante adaptação às mudanças ocorridas devido ao adoecer torna-se necessária16.

Pesquisa realizada por Bergamasco e Ângelo6 mostra a necessidade das pacientes adaptarem-se à nova condição de portadora de câncer de mama, até mesmo para conseguirem se ajustar às mudanças ocorridas após o diagnóstico.

Por sua vez, saber que tem uma doença sem causa definida traz ainda mais angústia e culpa. Vislumbrar 0 futuro passa a ser muito doloroso, já que os tratamentos propostos implicam em possível mutilação, náuseas, vômitos, alopecia, além de alterações sexuais e reprodutivas, como a menopausa precoce ${ }^{17}$.

Pollin ${ }^{18}$, em seu livro, descreve oito preocupações constantes na vida de pacientes que vivenciam doenças crônicas que ilustram claramente os maiores problemas trazidos também pelas mulheres com câncer de mama. As questões abordadas por ela são: perda do controle sobre a vida, mudanças na auto-imagem, medo da dependência, estigmas, medo do abandono, raiva, isolamento e morte. 0 medo da progressão da doença e da recidiva ${ }^{6}$ são outras preocupações constantes. 
D iversas pesquisas descrevem que ansiedade e depressão estão entre os problemas psicológicos mais freqüentes entre as pacientes com câncer $13,19,20$. Raminerz et al., citado por Carroll ${ }^{21}$, verificaram que $20 \%$ - 30\% das pacientes com câncer de mama têm ansiedade, depressão e baixa auto-estima em algum momento após 0 diagnóstico. Por sua vez, Carroll ${ }^{21}$ reforça que essas comorbidades podem continuar após o conhecimento do diagnóstico e até mesmo depois do término do tratamento.

Epping-J ordan et al. ${ }^{22}$ apontam fatores que podem estar associados com ansiedade e depressão durante 0 câncer de mama. Esses fatores são: variáveis demográficas, idade, nível educacional, estágio da doença, temperamento (otimista ou pessimista), respostas ao estresse e estratégias de confrontação com a doença.

0 sentimento de culpa, vivenciado por muitas mulheres que têm diagnóstico de câncer de mama, é outro fator abordado por Glinder et al. ${ }^{23}$ que está associado ao aumento da depressão e da ansiedade.

Estudo desenvolvido por Spiegel ${ }^{20} \mathrm{com}$ mulheres com câncer de mama, mostra que o grau da depressão independe da malignidade do tumor. D essa forma, só a suspeita de ter um câncer podetrazer abalos significativos na vida da mulher. Esse mesmo autor alerta que em diversos casos a depressão não é detectada, já que muitos dos seus sintomas, como falta de apetite e pouca energia, podem estar associados aos efeitos do tratamento ou às conseqüências do próprio câncer.

Baseado nos estudos de Simpson et al. ${ }^{24}$, outro problema psicológico muito comum entre os pacientes com câncer é o transtorno de ajustamento, atingindo $25 \%$ - 30\% dos pacientes.

Ao se estudar as conseqüências psicológicas causadas pelo câncer de mama é importante ressaltar que quanto maior a mutilação, mais traumático será o seu efeito ${ }^{19,25 .}$ $N$ esse caso, a mulher mastectomizada sofre seqüelas maiores, já que vivencia alterações significativas na sua imagem corporal.

Um medo muito freqüente entre as pacientes mastectomizadas é o de não ser mais atraente sexualmente. D essa forma, a presença do companheiro na reestruturação de sua integridade é fundamental. Porém, percebe-se que certas mulheres se afastam dos seus parceiros nesse momento, passando até a evitar contatos sexuais1. Em muitos casos, a mastectomia passa a ser um desafio para a relação homem-mulher.

É importante ressaltar que não apenas as mulheres que realizam a mastectomia vivenciam a baixa da autoestima. 0 próprio fato de estar com câncer, uma doença repleta de estigmas, já altera a percepção de si mesma, como mulher ${ }^{26}$.
Corroborando essa idéia, pesquisa desenvolvida por Alagaratnam e Kung, citada por M oyer ${ }^{25}$, afirma que há altos níveis de depressão e dificuldades sexuais tanto nas pacientes com câncer de mama tratadas com mastectomia quanto com as que fizeram cirurgias menos radicais. Esse estudo conclui que o diagnóstico de uma doença maligna é mais preocupante do que 0 desfiguramento da mastectomia.

Cabe mencionar que as reações das pacientes frente à doença, ao tratamento e à reabilitação dependem de características individuais, tais como: história de vida, contexto cultural e sociall ${ }^{27}$, espiritualidade e opção sexual ${ }^{28}$. Essas individualidades irão influenciar a forma de avaliar a importância da doença e a forma de enfrentála $a^{3,19}$.

Além das características individuais, diversas

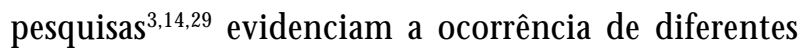
reações emocionais nas diversas etapas do tratamento, desde o diagnóstico até a recidiva. Percebe-se que mesmo com bons resultados do tratamento, a vivência do câncer freqüentemente deixa marcas na vida das mulheres ${ }^{14}$. M uitas delas passam a temer a ameaça do retorno da doença e a ter dificuldade em lidar com as seqüelas deixadas pelos tratamentos.

Todos esses fatores emocionais abordados, presentes na vida da mulher com câncer de mama, influenciam no sucesso do tratamento e da reabilitação, assim como em sua qualidade de vida ${ }^{19}$.

\section{ATUAÇÃO DO PSICÓLOGO: DE QUE FORMA O PSICÓLOGO PODE AJUDAR NESTE MOMENTO?}

\section{Breve histórico da Psicologia Oncológica}

As transformações no conceito de saúde, ocorridas entre os anos 70 e 90, quando passa a ser compreendida como um fenômeno biopsicossocial, constituíram um marco na criação de um espaço para o psicólogo nos Serviços de Saúde ${ }^{30}$.

O utro fato importante que contribuiu para a inserção do psicólogo na área de saúde foi a realização da Conferência Internacional sobre Cuidados Primários de Saúde, em 1978, na cidade de Alma-Ata, na então U nião Soviética, quando passou-se a enfatizar os cuidados primários, dando-se ênfase à promoção da saúde e prevenção da doença. A partir de então, ocorreu uma maior preocupação com a adesão do paciente ao tratamento. D essa forma, mais espaço se abre ao psicólogo, já que a adesão ou não ao tratamento muitas vezes implica em questões psicológicas ${ }^{30}$.

Antes da década de 70 , os poucos profissionais de saúde mental que trabalhavam na área oncológica atuavam em apenas três focos: pesquisas sobre 
personalidade e atitudes que poderiam causar o câncer; atendimento de problemas psiquiátricos dos pacientes com câncer causados pelo processo de ajustamento à situação e estudos sobre as questões da morte e luto ${ }^{4}$.

No final da década de 70, surgiram novos métodos de detecção precoce e tratamentos mais eficazes contra o câncer, resultando num aumento gradual da expectativa de vida do paciente e conseqüentemente numa maior preocupação com a sua qualidade de vida. Isso resultou no surgimento do campo de estudo e prática da Psicologia 0 ncológica 4 .

$\mathrm{N}$ a década de 80 , no Brasil, já se encontravam trabalhos isolados de pesquisa sobre assistência psicossocial a grupos de pacientes e de atendimento psicoterápico a pacientes em hospitais e consultórios, além da formação de grupos de auto-ajuda por pacientes e ex-pacientes de câncer. Estudos mostram que, desde então, várias pesquisas têm sido desenvolvidas nessa área, buscando formas de atuação cada vez mais eficientes 5 .

O Instituto N acional de Câncer / IN CA, órgão de referência nacional no tratamento dessa patologia, apenas em 1979 contratou o primeiro psicólogo. Em 1985 o Setor de Psicologia passa a ser um Setor independente, com um corpo formado por seis profissionais. Atualmente são doze profissionais atuando nas cinco unidades assistenciais*. Em 2003 esta instituição cria 0 primeiro curso de especialização em Psicologia O ncológica, formando dez profissionais.

Em 1998 obteve-se um grande avanço na área de psicologia, quando o M inistro da Saúde da época, através da portaria 3.535 do M inistério da Saúde, publicada no D.O.U. de 14/10/98, tornou obrigatória a presença de profissionais especialistas em Psicologia C línica como um dos critérios para cadastramento de Centros de Alta Complexidade em O ncologia (CACON) junto ao Sistema Ú nico de Saúde (SU S).

D essa forma, o psicólogo passa a ser membro da equipe multidisciplinar cuidadora do paciente com câncer, atuando em todas as etapas do processo do tratamento oncológico.

\section{Papel do psicólogo oncológico}

0 psicólogo atuante na área de psicologia oncológica visa manter o bem-estar psicológico do paciente, identificando e compreendendo os fatores emocionais que intervêm na sua saúde. 0 utros objetivos do trabalho desse profissional são prevenir e reduzir os sintomas emocionais e físicos causados pelo câncer e seus tratamentos, levar o paciente a compreender 0 significado da experiência do adoecer, possibilitando assim re-significações desse processo ${ }^{3,16}$.

Em sua atuação, o psicólogo deve estar atento também aos distúrbios psicopatológicos, como depressão e ansiedade graves.

Sua prática é exercida em todas as etapas do tratamento, como dito anteriormente, habilitando 0 paciente a confrontar-se com o diagnóstico e com as dificuldades dos tratamentos decorrentes, ajudando-0 a desenvolver estratégias adaptativas para enfrentar as situações estressantes.

O s objetivos do trabalho do psicólogo oncológico serão alcançados na medida em que esse profissional vai compreendendo o que está envolvido na queixa do paciente, buscando sempre uma visão ampla do que está se passando naquele momento não escolhido da vida del $\mathrm{e}^{16}$.

$\mathrm{N}$ um espaço de acolhimento e escuta o terapeuta deve sempre trabalhar com a realidade. $\mathrm{Q}$ uanto mais informado o paciente estiver de sua doença, maior será a sua capacidade de enfrentar 0 adoecer e mais confiança terá na equipe. Pacientes bem informados reagem melhor ao tratamento ${ }^{13}$. D essa forma, o psicólogo deve preocupar-se em falar numa linguagem acessível ao paciente e sempre checar se as informações e orientações dadas pela equipe foram efetivamente compreendidas ${ }^{16}$.

Segundo Ferreira ${ }^{12}$, os temas que mais preocupam os pacientes com câncer e que devem ser focos das intervenções psicoterápicas são: sensação de falta de controle sobre a própria existência, temor da solidão e da própria morte, sentimento de impotência e fracasso e temor dos efeitos adversos do tratamento oncológico.

A atuação do psicólogo não se restringe ao paciente oncológico. É imprescindível incluir a família no atendimento ${ }^{31}$, já que são personagens fundamentais no auxílio aos pacientes para o enfrentamento da doença. $\mathrm{N}$ a medida em que tenha suporte, a família pode se tornar aliada permanente não só do paciente, como também da própria equipe de saúde $e^{12,16}$.

Pesquisa realizada por Bergamasco e Ângelo6 afirma que a família, com sua ajuda material e emocional, é considerada pelas mulheres com câncer de mama como sendo um apoio fundamental para darem continuidade ao tratamento.

Para Simpson et al. ${ }^{24} 0$ suporte social também é um fator importante na prevenção e alívio da depressão e ansiedade nos pacientes com câncer. N este artigo, esses autores citam uma pesquisa com pacientes com câncer de mama mostrando que a ansiedade e a depressão, três meses após a cirurgia, estão relacionadas com 0

* D ados colhidos por psicólogos dessa instituição. 
grau de suporte dos membros da família e do cirurgião.

Atuando junto à família, o psicólogo deve buscar reforçar os vínculos afetivos entre família e paciente, facilitando um diálogo verdadeiro, capacitando-os a compartilhar experiências e emoções. A participação dos familiares nas decisões, junto ao paciente, constitui outro fator que deve ser estimulado pelo psicólogo ${ }^{16}$.

É importante mencionar que o psicólogo oncológico trabalha numa rede de contatos, ou seja, não trabalha sozinho e sim com a equipe de saúde. A interação entre todos os profissionais envolvidos no tratamento do câncer de mama é fundamental para a conquista de um bom resultado, já que a atuação passa a ser global, envolvendo todos os aspectos implicados no adoecimento.

0 psicólogo deve estar atento aos focos de conflitos dentro da equipe de saúde, impedindo ruídos que possam surgir nessa comunicação, pois é a partir da relação desses profissionais que todas as demais relações podem viabilizar-se ou se comprometerem ${ }^{16}$.

Para que a atuação do psicólogo junto à equipe e ao paciente se desenvolva satisfatoriamente, é necessário que esse profissional tenha informações básicas sobre 0 câncer, além dos tratamentos e sintomas que resultam dos mesmos. É importante para o psicólogo saber distinguir sintomas emocionais dos sintomas decorrentes das questões orgânicas. 0 conhecimento básico das questões médicas referentes ao câncer de mama, como, por exemplo, os estágios da doença e prognósticos associados a esses, contribui para que o psicólogo possa definir a abordagem que irá utilizar com o paciente.

\section{Possibilidades de atuação do psicólogo no tratamento de pacientes oncológicos}

0 psicólogo oncológico trabal ha tanto na instituição hospitalar quanto na sua própria clínica. 0 aporte teórico-técnico utilizado deve ser articulado conforme o local onde se desenvolve o trabalho, o diagnóstico do paciente, 0 tipo de intervenção e principalmente 0 objetivo que pretende ser alcançado ${ }^{32}$.

0 psicólogo que trabalha com pacientes portadores de doenças crônicas deve ter uma postura mais ativa, com um foco no adoecimento e nas preocupações do momento, visando sempre um atendimento humanizado e global ao doente ${ }^{16}$.

Para Penna ${ }^{33}$, esse tipo de psicoterapia dever ter como objetivo "melhorar, modificar e atenuar aquilo que é disfuncional e que cause sofrimento ao paciente, que 0 impeça de utilizar formas adaptativas para lidar com a patologia orgânica, visando uma melhora na qualidade de vida do indivíduo na vigência de uma doença".
Técnicas de relaxamento, como a auto-hipnose ou o relaxamento progressivo, são muito utilizadas com pacientes oncológicos, já que permitem que o mesmo consiga ter um maior controle sobre suas emoções e sintomas, como, por exemplo, saber controlar o pânico, a dor, náusea e vômitos ${ }^{20}$. 0 sentimento de impotência é muito presente entre os pacientes, já que nada pode ser feito para evitar um câncer, havendo também desconhecimento sobre como será o desenvolvimento da doença. Por isso, essa sensação de ter algum controle sobre os sintomas decorrentes da doença ou tratamentos é muito positiva para o paciente, pois se vê capaz de fazer algo em seu próprio benefício ${ }^{33}$.

0 grupo é outro tipo de intervenção muito utilizado na prática da psicologia oncológica. A literatura especializada traz fortes evidências da eficácia da terapia de grupo com pacientes com câncer.

M elo Filho ${ }^{32}$, citando Irvin Yalom, define algumas características exclusivas dos grupos psicoterápicos que contribuem para sua eficácia: compartilhar informações, universalidade de conflitos, altruísmo, comportamento identificativo, aprendizagem interpessoal, coesão grupal e catarse.

$\mathrm{N}$ a prática existem diferentes abordagens grupais que se diferem em sua finalidade, tais como: ensinoaprendizagem, comunitário, terapêutico, psicodramático, da teoria sistêmica, cognitivo-comportamental e psicanalítico34. A escolha de qual será a melhor abordagem a ser utilizada deve ser calcada no tipo de grupo (de ambulatório ou de enfermaria, homogêneo ou heterogêneo, aberto ou fechado), sobre o objetivo (breve, de médio ou longo prazo) e sobre outras variáveis (informativo, de preparo para cirurgia ou para terapia, etc.). Pesquisas evidenciam que para aumentar a eficácia dos grupos, eles devem ter metas, estrutura e métodos de intervenção feitos sob medida para suas características específicas ${ }^{32}$.

Segundo M ello Filho ${ }^{32}$, nos grupos de pacientes somáticos, as interpretações psicanalíticas, ou seja, interpretações dirigidas a aspectos inconscientes, são contra-indicadas. I sso porque o paciente já se encontra sobrecarregado com a doença somática, com a auto-estima baixa, frágil, propenso a depressões, regressões e atitudes auto-agressivas. $\mathrm{N}$ este caso, esse mesmo autor reforça que é mais indicado utilizar interpretações prévias como: clarificação, assinalamento econfrontação, estando sempre atento ao que o paciente possa tolerar.

As práticas atuais de terapia de grupo privilegiam os grupos homogêneos, ou seja, pacientes que tenham alguma identificação entre si ${ }^{32}$. $\mathrm{N}$ a abordagem deste trabalho, a homogeneidade se dá pela patologia. O wen et al..$^{35}$ evidenciam que quanto mais homogêneo 0 grupo 
for, melhores serão os objetivos alcançados e mais focal será a intervenção.

Para M ello Filho ${ }^{32}$ reunir pacientes com a mesma patologia num grupo só traz vantagens, já que permite ao paciente perceber melhor seus problemas, vendo esses nos outros, aprendendo a tolerar o que repudiam em si, melhorando assim a resolução da doença.

Segundo Spiege ${ }^{20}$, o trabalho de grupo é uma forma de criar relações sociais, fato importante já que muitos pacientes tendem a se isolar e a se sentir rejeitados. Entre iguais, no grupo, os pacientes se sentem aceitos, proporcionando, assim, uma melhor integração entre os participantes.

$H$ abeet al. ${ }^{11}$ ressaltam que além de ser uma prática terapêutica que traz ótimos resultados, a terapia de grupo permite um tratamento mais viável economicamente, se comparada ao custo da terapia individual.

$\mathrm{Na}$ literatura internacional verifica-se que grande parte das pesquisasutiliza a abordagem cognitivo-comportamental para as intervenções com os pacientes com câncer. Segundo Penna ${ }^{33}$, nos países anglo-saxões essa realmente é a técnica mais utilizada. Já no Brasil as "terapias expressivas" são as mais usadas. Essas terapias buscam a compreensão do significado simbólico da doença e dos conflitos emocionais inconscientes ligados ao adoecimento, não dando muita ênfase na mobilização dos recursos cognitivos para se adequar melhor à situação.

Pesquisas mostram a dificuldade de determinar que tipo de método é mais apropriado para trabal har com 0 paciente com câncer ${ }^{35,36}$. Entretanto, acredita-se que a abordagem teórico-técnica utilizada serve de embasamento para o desenvolvimento do trabalho e não como determinante de sua eficácia. 0 que todos devem ter em comum são os objetivos que deverão ser alcançados, utilizando a abordagem com a qual 0 psicólogo melhor se identifique.

É importante mencionar que antes de começar qualquer atendimento psicoterápico é preciso detectar se 0 paciente realmente precisa de uma intervenção especializada e se há uma demanda para isso ${ }^{33}$.

\section{CONSEQÜÊENCIAS POSITIVAS DA ATUAÇÃO DO PSICÓLOGO}

A literatura especializada mostra que pacientes submetidos ao acompanhamento psicológico durante 0 tratamento do câncer de mama obtêm ganhos significativos, tais como: melhora do estado geral de saúde; melhora da qualidade de vida, melhor tolerância aos efeitos adversos da terapêutica oncológica (quimio/ radioterapia e cirurgia) e melhor comunicação entre paciente, família e equipe ${ }^{20,37}$. D essa forma, percebe-se que as intervenções psicológicas podem ter conseqüências positivas tanto no aspecto emocional (depressão e ansiedade) quanto nos sintomas físicos (náuseas, vômitos e fadiga) ${ }^{20}$.

Pesquisas mostram que as intervenções psicossociais influenciam positivamente 0 ajustamento emocional e funcional da paciente e aliviam os sintomas adversos decorrentes do câncer e do seu tratamento ${ }^{35,36}$.

$O$ utra conseqüência de um bom trabalho psicoterápico é a participação mais ativa e positiva da paciente durante o tratamento, resultando numa melhor adesão, evitando assim, 0 abandono do mesmo. Estudos evidenciam que quando a paciente encontra-se mais participativa durante o tratamento, há menor probabilidade do surgimento de intercorrências clínicas e psicológicas no mesmo ${ }^{38}$.

Além desses resultados que o psicólogo busca em sua atuação, poderão ocorrer efeitos adicionais como 0 aumento do tempo de sobrevida da paciente ${ }^{20,37,39,40}$.

Cohen e Lazarus, citados por Gimenez ${ }^{3}$, propõem que a longevidade da paciente com câncer está relacionada com a capacidade de ajustamento do indivíduo à doença. Esta mesma autora enfatiza outros fatores que influenciam na sobrevida da paciente, tais como: expressão dos sentimentos, vontade de viver, reação ativa em relação ao tratamento e bom suporte social e afetivo. Fatores estes incentivados no trabalho psicoterápico.

D iversas pesquisas ${ }^{20,37}$ com pacientes portadoras de câncer de mama demonstram que as pacientes que se submetem a intervenções psicológicas além dos tratamentos médicos habituais possuem um diferencial qual itativo e quantitativo em sua saúde comparativamente àquelas que não participam desse tipo de atividade, aumentando significativamente seu tempo de vida.

Entre os vários trabal hos referentes a este tema, destaca-se a pesquisa desenvolvida por Spiegel ${ }^{20,41}$ sobre o efeito da participação de pacientes com câncer de mama com metástase, numa psicoterapia de grupo. As sessões realizadas tinham como objetivo incentivar a expressão dos sentimentos sobre a doença, estimular 0 desenvolvimento de fortes vínculos entre as participantes, além de lhes ser ensinado hipnose para controle da dor. O s resultados mostram redução significativa nos transtornos de humor, além de obter efeitos positivos em relação ao enfrentamento da doença e da dor ${ }^{20}$.

Baseado nessa mesma pesquisa, Spiegel ${ }^{41}$ constatou que além do efeito benéfico sobre o humor e 0 vigor das pacientes alcançados com a intervenção psicoterapêutica, as pacientes tiveram um aumento significativo em sua sobrevida, comparando-se com 0 grupo de controle. Para esse mesmo autor, esse último fato foi resultado, entre outros motivos, dos efeitos positivos da melhora dos estados emocionais sobre a 
atividade dos sistemas imunológico e neuroendócrino, interferindo na progressão do câncer.

Estudos recentes têm comprovado cada vez mais que as perturbações emocionais prejudicam 0 bom funcionamento do sistema imunológico causando alterações bioquímicas, constituindo assim um dos fatores responsáveis pela origem e desenvolvimento das doenças ${ }^{19,38,42,43}$. D essa forma, a paciente, não estando bem emocionalmente, estará mais vulnerável para adoecer.

I númeras pesquisas demonstram a associação de certos fatores psicológicos à origem e prognóstico do câncer. O s fatores mais recorrentes na literatura são: estados afetivos, especialmente depressão; eventos de vida estressantes, com destaque para as perdas; apoio social; personalidade e estilos de enfrentamento das situações problemáticas ${ }^{37,42,43}$.

Pesquisas ${ }^{37,42}$ sobre a influência das reações psicológicas ao diagnóstico de câncer de mama na sobrevida das pacientes demonstram uma significativa ligação entre o prognóstico e essas reações. As pacientes que se enquadram no tipo de reação denominada "espírito de luta", ou seja, determinadas a lutar ativamente contra a doença, adotando uma atitude otimista, tiveram maior sobrevida do que aquelas que reagiam com sentimentos de desesperança e desamparo.

A influência dos fatores psicológicos sobre os aspectos biológicos é demonstrada através de estudos ${ }^{37,39}$ com um tipo de célula produzida pelo sistema imunológico, a célula natural killer (N K). Eles mostram uma associação entre algumas variáveis psicossociais e o funcionamento dessas células. O s mesmos trazem evidências de que um baixo nível de ajustamento ao câncer, falha no apoio social, alto nível de estresse e presença de sintomas depressivos diminuem a produção e atividade da NK. Sendo essa célula responsável pela vigilância imunológica sobre o câncer, controlando a difusão das células malignas, a paciente tenderá a ter um pior prognóstico.

D e acordo com esses dados, conclui-se que o sistema imunológico é fortemente afetado por fatores emocionais e que determinadas atitudes psicológicas podem influenciar positivamente no sistema de defesa, favorecendo uma maior e melhor sobrevida. M esmo com todos os avanços nessa área de estudo, ainda há muitas questões a serem esclarecidas e comprovadas44.

Esse resultado adicional da intervenção psicológica no aumento da sobrevida da paciente também está associado tanto aos hábitos mais saudáveis, que a mesma incorpora em sua vida, como a sua adesão aos tratamentos. Isso ocorre porque a intervenção psicológica afetará positivamente 0 estado emocional da paciente motivando-a a adotar esses comportamentos ${ }^{38}$.
Existem autores ${ }^{35,45}$ que questionam a validade de certas pesquisas que analisam a eficácia das intervenções psicológicas sobre 0 aumento da sobrevida e sobre 0 estado emocional nos pacientes com câncer, já que existem diversas variáveis difíceis de serem controladas. M esmo não dando crédito a certos dados científicos, Cunningham et al..$^{45}$ afirmam perceber em suas práticas que os pacientes envolvidos em tratamento psicológico vivem mais do que as expectativas médicas.

$\mathrm{N}$ ão são apenas os pacientes e familiares que se beneficiam com 0 atendimento psicológico. As instituições de saúde também lucram, já que a ampliação do âmbito da assistência resulta na diminuição do uso de serviços médicos em geral, reduzindo o tempo de hospitalização, conseqüentemente caindo os custos hospitalares. M artins31 cita um importante estudo metaanalítico realizado por $M$ umford et al. que revela evidências na redução do custo do uso de serviços médicos após intervenções psicossociais. Os trabal hos analisados resultaram nas seguintes observações: redução no tempo da internação, diminuição de $20 \%$ no uso de serviços médicos e queda nas solicitações de exames. Verificou-se também que o custo de um tratamento psicoterápico era compensado pela economia que se obtém através da diminuição do uso de serviços médicos. N estemesmo texto, $M$ artins cita outro trabal ho desenvolvido por Strain et al. que demonstra ter havido uma redução de custos hospitalares de até 160.000 dólares devido à diminuição do tempo de hospitalização após intervenções psicossociais.

Todos os avanços obtidos no âmbito da Psicologia O ncológica têm influenciado um maior reconhecimento por parteda comunidadecientífica do trabalho do psicólogo com pacientesoncológicos. A American Cancer Society, citada por O wen ${ }^{35}$, conclui que:

"Como muitos estudos bem controlados indicam que grupos de apoio apropriadamente planejados e supervisionados melhoram a qualidade de vida dos pacientes com câncer, a American Cancer Society os encoraja como um componente valioso e altamente compensador em serviços psicossociais no tratamento do câncer".

M esmo com certos reconhecimentos e com todas as evidências descritas no campo científico e observadas na prática, dos benefícios do atendimento psicológico ao paciente com câncer, esse serviço ainda não foi incorporado nas rotinas de assistência desse paciente ${ }^{36}$.

Segundo 0 wen ${ }^{35}$, para que isso aconteça será necessário, primeiro, convencer os outros profissionais da área de saúde que, além da melhora na qualidade de vida do paciente, há também benefícios financeiros com 0 atendimento psicológico. 


\section{CONCLUSÃO}

A partir do levantamento da literatura especializada, aliado à experiência com atendimentos às pacientes com câncer de mama, constata-se a relevância do trabalho do psicólogo junto a essas mulheres.

A atuação desse profissional deve se adequar à demanda que lhe é feita, traçando intervenções que possam ter efeitos positivos no enfrentamento da doença e dos tratamentos. Sua prática visa possibilitar a mulher com câncer de mama uma melhor qualidade de vida.

Atualmente, percebe-se uma maior inserção do psicólogo na área da Saúde. Porém, muitas conquistas ainda podem ser feitas buscando uma maior integração entre os diversos profissionais da área de Saúde, visando a criação de um espaço próprio para a atuação do psicólogo.

D essa forma, acredita-se ser importante uma maior congregação entre os psicólogos hospitalares atuantes na área oncológica, ampliando os espaços de trocas e de produção científica. A valorização dos cursos de especialização nessa área é outro fator importante para a formação de profissionais capacitados para a realização de um bom trabalho.

Essas sugestões propostas buscam facilitar a divulgação da relevância do trabalho do psicólogo no âmbito da oncologia a fim de criar uma demanda e reconhecimento para esse profissional.

\section{REFERÊNCIAS BIBLIOGRÁFICAS}

1. Arán M R, Zahar S, D elgado PG, V iegas $M, C$ abral CP, Souza CM, et al. Representações de pacientes mastectomizadas sobre doença e mutilação e seu impacto no diagnóstico precoce do câncer demama. J BrasPsiquiatr 1996;45(11):533-9.

2. Gazzi G, Kajika M , Rodrigues C. 0 pacientecom câncer: crenças e sentimentos sobre sua doença e o tratamento. Acta O ncol Bras 1991;11(1/3):123-6.

3. Gimenez M G, organizador. A mulher eo câncer. São Paulo: Editorial Psy; 1997. p. 325.

4. Leal VM . H istórico dainserção da psicologiano tratamento oncológico [palestra]. Instituto $\mathrm{N}$ acional deC âncer. H ospital do Cancer III. set 2001.

5. Carvalho M M , organizador. Psico-oncologia no Brasil: resgatando o viver. São Paulo: Summus; 1998. p. 256.

6. Bergamasco R, Angelo M . 0 sofrimento de descobrir-se com câncer demama: como o diagnóstico éexperienciado pela mulher. Rev BrasC ancerol 2001;47(3):227-82.

7. Parkin D M , Bray FI, D evesaSS. C ancer burner in theyear 2000. The G lobal Picture. Eur J C ancer 2001;37:S4-S66.
8. Instituto $\mathrm{N}$ acional de $\mathrm{C}$ âncer (Brasil). Estimativas da incidência emortalidade por câncer 2003. Rio deJ aneiro: IN CA; 2003. p. 29.

9. Instituto $\mathrm{N}$ acional de Câncer (Brasil). Estimativas da incidência emortalidade por câncer 2001. Rio deJ aneiro: IN CA; 2001. p. 25.

10. K oifman S, K oifman R. Incidênciaemortalidadepor câncer em mulheres adultas no Brasil. In: Giffin K, Costa S, organizadores. Q uestões da saúde reprodutiva. Rio de Janeiro: Fiocruz; 1999.

11. H aber S, Ayers L, G oodheart C, Lubin L, Siegel M , Acuff $C$, et al. Breast cancer: a psychological treatment manual. $\mathrm{N}$ ew York: Springer; 1995. p. 138.

12. Ferreira PE. Alguns pacientes especiaisno hospital geral: 0 pacienteoncológico. Cad IPUB 1997;6:143-54.

13. SalesC, PaivaL, Scandiuzzi D, Anjos AC. Q ualidadedevida de mulheres tratadas de câncer de mama: funcionamento social. Rev Bras C ancerol 2001;47(3):263-72.

14. Bloom J. Surviving and thriving? Psycho-O ncology 2002;11:89-92.

15. Parker P, Baile W, M oor C, Cohen L. Psychosocial and demographic predictors of qualiyt of life in a largesample of cancer patients. Psycho-O ncology 2003;12:183-93.

16. SantosC, Sebastiani R. A companhamento psicológico àpessoa portadoradedoençacrônica. In: Angerami-Camon V, Chialtone $H$, Sebastiani R, Fongaro M L, SantosC. E apsicologiaentrou no hospital. São Paulo: Pionera; 1996. p. 147-76.

17. N etto M . 0 tratamento do câncer demamaea psicologia: um foquemultidisciplinar. Psyche 1997;1(1):165-9.

18. Pollin Irene. M edical crisiscounseling: short- term therapy for long-term illness. N ew York: W.W. N orton; 1995. p. 208.

19. Guntert AE. Contribuiçõesda psicológica parao tratamento e reabilitação do paciente com câncer. Acta O ncol Bras 1995;15(2):84-8.

20. Spiegel $D$. Facilitating emotional coping during treatment. Cancer 1990;66 Suppl 15:1422-6.

21. C arrol S. Psychological responseand survival in breast cancer. Lancet 2000;335:404-6.

22. Epping-J ordan J, C ompas B, O sowiecki D , 0 ppedisano G, G erhardt C, Primo K, et al. Psychological adjustment in breast cancer: processes and emocional distress. $\mathrm{H}$ ealth Psychol 1999;18(4):315-26.

23. G linder J, CompasB. Self-blameattributionsin women with newly diagnosed breast cancer: a prospective study os psychological adjustment. H ealth Psychol 1999;18(5):475-81.

24. Simpson E, C arlson L, Beck C, Patten S. Effects of a brief intervention on social support and psychiatric morbidity in breast cancer patients. Psycho-O ncology 2002;11:282-94.

25. M oyer Anne. Psychosocial outcomes of breast-conserving surgery versusmastectomy: a meta-analytic review. $\mathrm{H}$ ealth Psychol 1997;16(3):283-98.

26. Bertero CM . Affected self-respect and self-value: theim- 
pact of breast cancer treatment on self-esteem and Q ql. Psycho-O ncology 2002;11:356-61.

27. Ashing K, Padilla G, Tejero J, Kagawa-Singer M . Understanding the breast cancer experience of Asian American Women. Psycho-O ncology 2003;12:38-58.

28. Fobair P, O ' H anlan K, Koopman C, Classen C, D imicele $\mathrm{S}, \mathrm{D}$ rooker $\mathrm{N}$, et al. Comparison of lesbian and heterosexual women'sresponseto newly diagnosed breast cancer. Psycho-O ncology 2001;10:40-51.

29. Frost M , Suman $V$, Rummans T, D ose AM , Taylor $M$, N ovotny P, et al. Physical, psychological and social wellbeing of women with breast cancer: the influence of disease phase. Psycho-O ncology 2000;9:221-31.

30. M uylaert M A. C orpoafecto: o psicólogo no hospital geral. São Paulo: Escuta; 1995. p. 111.

31. M artins LA. A interconsulta como instrumento da psiquiatria dehospital ização. Saúdemental no hospital geral. Cad IPUB 1997;6:33-44.

32. M ello Filho J. G rupo ecorpo: psicoterapia degrupo com pacientes somáticos. São Paulo: Artmed; 2000. p. 435.

33. PennaT. Psicoterapia no hospital geral. Saúdemental no hospital geral. Cad IPU B 1997;6:195-204.

34. Zimerman D, O sório LC. Como trabal hamos com grupos. Porto Alegre: Artes M édicas; 1997. p. 424.

35. O wen J, Klapow J, H icken B, Tucker D . Psychosocial interventionsfor cancer: review and analysis using a threetiered outcomes model. Psycho-O ncology 2001;10:218-30.

36. M eyer T, M ark M. Effects of psychosocial interventions with adult cancer patients: a meta-analysis of randomized experiments. H ealth Psychol 1995;14(2):101-8.

37. Leal V. Variáveis psicológicas influenciando o risco e 0 prognóstico do câncer: um panorama atual. Rev Bras Cancerol 1993;39(2):53-9.

38. C arvalho V. Aspectos psicológicosligadosao pacientecom câncer earelação médico-paciente. In: C oelho FR. C urso básico de oncologia. São Paulo: M edsi; 1996. p.165-76.

39. Amorim AM . A enfermagem eapsiconeuroimunologiano câncer demama [dissertação]. Rio deJ aneiro (RJ): Escolade Enfermagem Anna N ery; 1999. p. 142

40. Rezende V, Botega N. Grupo de apoio psicológico a mulheres com câncer de mama: principais fantasias inconscientes. Estud Psicol 1998;15(1):39-48.

41. Spiegel D, Bloom Jr, Kraemer H C, Gotthil E. Effects of psychosocial treatment on survival of patientswith metastic breast cancer. Lancet 1989;2(2):888-91.

42. Watson M, H aviland J, Greer S, D avidson J, Bliss J . Influence of psychological response on survival in breast cancer: a population-based cohort study. Lancet 1999;354:1331-6.

43. Leshan L. 0 câncer como ponto demutação: um manual para pessoas com câncer, seusfamiliares e profissionais de saúde. 3a ed. São Paulo: Summus; 1992. p. 195.

44. G laser J, G laser R. Psychoneuro immunology: past, present, and future. H ealth Psychol 1989;8(6):677-82.

45. Cunningham A, Edmonds C, Phillips C, SootsK, H edley $D$, Lockwood G. A prospective, longitudinal study of the relationship of psychological work to duration of survival in patients with metastatic cancer. Psycho-0 ncology 2000;9:323-39. 